\title{
Michel Butor oder ein virtuelles Bereisen unseres Planeten
}

Der vor wenigen Jahren verstorbene Michel Butor darf wohl als einer der scharfsinnigsten und zugleich spielerischsten Autoren der zweiten Hälfte des 20. und der ersten Jahrzehnte des 21. Jahrhunderts gelten. Wie vielleicht nur noch bei Jean-Marie Gustave Le Clézio steht bei ihm das Reisen im Zentrum allen Schreibens, ja mehr noch: ist das Reisen selbst bereits eine Form des Schreibens. Beschäftigen wir uns kurz mit der Lebensreise dieses überaus sympathischen Schriftstellers!

Michel Marie François Butor wurde an einem 14. September des Jahres 1926 in Mons-en-Barœul in der Nähe von Lille geboren und verstarb am 24. August 2016 in Contamine-sur-Arve. Michel war das vierte Kind des Eisenbahninspektors Emile Butor und seiner Frau Anne Brajeux, die in einem Vorort der französischen Stadt Lille ihre Kinder großzogen. Als begabter Schüler besuchte er das Jesuitenkolleg Saint François-de-Sales in Evreux und das renommierte Gymnasium Louisle-Grand in Paris. Schon während der Gymnasialzeit las er viel, darunter Werke von Paul Claudel, Marcel Proust und Franz Kafka. In diese Zeit fielen auch seine ersten dichterischen Versuche. Doch wie sein Vater zeichnete er gerne und wollte, wie er später schrieb, zunächst Maler werden, bevor er sich dann doch für die Schriftstellerei entschied. Butor hat sich Zeit seines Lebens eine große Affinität zur Malerei bewahrt, wovon sein schöner Buch-Essay Les mots dans la peinture (Die Worte in der Malerei), aber auch zahlreiche Kunstkritiken und Auseinandersetzungen mit großen Werken der Kunstgeschichte künden.

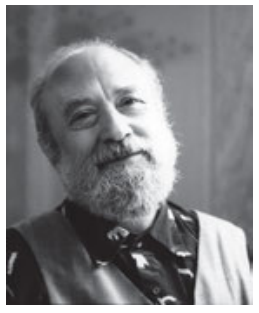

Abb. 103: Michel Butor (Mons-en-Barœul, 1926 - Contamine-sur-Arve, 2016).

Ab 1944 begann er ein Studium der Literatur, Philosophie und Philologie an der Pariser Sorbonne, an der er 1949 mit einer Arbeit über Mathematik und Ästhetik promovierte. Nach seinem Studium arbeitete er als Lehrer und danach als Französisch-, Latein- und Philosophielektor in Sens, in Ober-Ägypten, Manchester, Thessaloniki und Genf, bevor er 1956/57 Professor für Französisch, Geschichte und Geographie an der Ecole Internationale in Genf wurde, einer Stadt, mit der 
ihn eine besondere Beziehung verband. Ab 1960 war er Lektor in den USA, unter anderem in Middlebury, Buffalo, Evanston und Albuquerque, später auch in Main, dann in Tokio und schließlich in Kanada. Seit 1958 lektorierte Butor zudem beim französischen Verlag Gallimard und unterrichtete seit 1970 französische Literatur an der Universität Nizza. Ab 1975 Lehrstuhlinhaber in Genf für moderne französische Literatur, war er dort auch nach seiner Emeritierung im Jahr 1991 tätig.

Michel Butor verband seine Arbeit mit dem Reisen, reiste jedoch daneben auch sehr häufig aus privater Leidenschaft, wobei ihn Reisen unter anderem 1951 nach Tunis, 1958 nach Venedig, 1963 nach Bulgarien und ins damalige Jugoslawien, 1966 nach Japan, 1967 in die Sowjetunion, 1971 nach Neuseeland und Australien führten. 1964 und 1965 lebte er auf Einladung der Ford Foundation in Berlin. Man kann ohne Übertreibung sagen, dass Michel Butor ebenso passionierter Reisender wie passionierter Schriftsteller war, der beide Tätigkeiten stets miteinander und jenem lächelnden Humor verband, der für diesen Autor charakteristisch war. Er gehörte $1960 \mathrm{zu}$ den Mitunterzeichnern des Manifests der 121 gegen den französischen Algerienkrieg.

Michel Butor darf als einer der großen Vertreter des Nouveau Roman gelten: Seine Romane L'Emploi du temps (Der Zeitplan, 1956) und vor allem La Modification (Die Modifikation, 1957) wurden mit zahlreichen Preisen bedacht und gelten bis heute als herausragende Repräsentanten einer neuen „écriture“. Für letztere stehen stellvertretend Autorinnen und Autoren wie Alain Robbe-Grillet oder Nathalie Sarraute, mit der wir uns noch beschäftigen werden. Im Verlauf der sechziger Jahre distanzierte sich Butor eher leise und dezent vom Nouveau Roman und wandte sich anderen experimentellen Schreibformen zu. Ihm gewidmete Kolloquien zementierten unterdessen die Zurechnung zum „Neuen Roman“ Frankreichs, wobei Butor nun auch dank seiner Essays als einer der führenden Köpfe dieser literarischen Strömung galt. Stets erachtete er den Roman als experimentelle Form und Labor für neue Sichtweisen unserer Welt, stand aber in einer Vielzahl von Gattungen für die Literatur als Laboratorium menschlicher Kreativität ein.

Seine Frau und seine vier Töchter hielt der Schriftsteller stets aus allen Pariser Wirbeln heraus und zog seit 1987 ins französische Lucinge in der Nähe von Genf. Sein Werk wurde mit einer Vielzahl von Auszeichnungen bedacht und darf auch in literaturtheoretischer Hinsicht als eines der einflussreichsten der zweiten Hälfte des 20. Jahrhunderts gelten. Seine fundamentalen Einsichten in die Verbindungen der Narration zu Raum und Zeit entwickelten sich zweifellos vor dem Hintergrund all seiner Reisen, die für ihn stets in einem essentiellen Zusammenhang mit dem Schreiben standen. Dabei waren es ebenso reale wie virtuelle Reisen, die sich in zunehmendem Maße zu einer ganzen Welt, einem vollständigen Planeten 
rundeten, mit dem wir uns zu Beginn unserer Beschäftigung mit diesem französischen Schriftsteller auseinandersetzen wollen.

In seinen ebenso hintergründigen wie humorvollen Paysages planétaires entfaltete Butor in poetisch verdichteter Form Landschaften, in denen sich die unterschiedlichsten Teile unseres Planeten miteinander verbinden und sich in ständiger, unablässiger Bewegung befinden. So widmet sich gleich der Beginn einer hybriden Komposition mit dem Titel ALASKAMAZONIE, ${ }^{1}$ die allseits von Leben erfüllt ist:

\author{
Die Wipfel der Koniferen \\ das Reich der Raben \\ der Große und der Kleine Bär \\ die borealen Dämmerungen \\ die Reste der Goldsucher \\ die Schlitten auf der Tundra \\ die genealogischen Masten \\ das Kupfer und die Walrosszähne
}

Das Meer, Gezeiten und Einfaltungen, mit den Schreien der Möven, weit und Ebbe und Fluten, mit den Gesängen der Wale weit weg. Durch die Fenster des Schiffes sehen wir, wie Fjorde und Gletscher vorbeiziehen. Plötzlich lösen sich Blöcke und treiben spritzend in den Kanälen. Jetzt kommen die Jäger, die mit Fleisch und Pelzen heimkehren.

Das Reich der Kolibris
die borealen Dämmerungen
die zerstörerischen Zyklonen
die schlitten auf der Tundra
die Flöße auf den Strömen
das Kupfer und die Walrosszähne
die Lichtkegel der Gefieder
das Reich der Raben ${ }^{2}$

Flora und Fauna hoher und niedriger Breiten, ${ }^{3}$ Tundra und Tropen, Land und Meer, Hitze und Kälte durchdringen sich wechselseitig, ohne miteinander zu

1 Butor, Michel: Paysages planétaires. In (ders.): Seize lustres. Euvres complètes. Bd. XII: Poésies 3 (2003-2009). Paris: La Différence 2010, S. 738.

2 Ebda.

3 Vgl. hierzu auch die beiden Arbeiten von Suter, Patrick: Butor et le livre-installation - montage de textes, œuvre plurielle, transits entre univers culturels. In: Weiand, Christof (Hg.): Les graphies du regard. Die Graphien des Blicks - Michel Butor und die Künste. Heidelberg: Universitätsverlag Winter 2013, S. 43-61; sowie ders.: Butor transaréal. In: Biglari, Amir / Desoubeaux, Henri (Hg.): Dix-huit lustres. Paris: Classiques Garnier 2016, S. 423-445. 
fusionieren, in Bewegungen von weltumspannenden Ausmaßen, die hier den amerikanischen Kontinent ineinander führen, in anderen Teilen der Paysages planétaires aber auch die Kontinente in einen wechselseitigen Austausch treten lassen. Alles ist in diesen Bildern in Bewegung.

Meeres- und Flusslandschaften beleuchten sich wechselseitig vermittels des sie verbindenden, lebensspendenden Wassers. Wie in Butors epochalen experimentellen Text Mobile - mit dem wir uns sogleich auseinandersetzen werden - ist hier alles über die unterschiedlichsten Transportmittel miteinander verbunden und in einem wechselseitigen Transformationsprozess begriffen, der die Menschen, Tiere, Pflanzen, Gesteine, Winde, Wasser und Gefieder erfasst. Nichts auf diesem Planeten steht für sich allein. Michel Butor zeigt uns eine Welt, in der alles Wechselwirkung ist.

In rascher Folge durchquert seine Leserschaft - um hier nur einige Titel zu nennen - die ETATS ZUNI $I^{4}$ oder die VIETNAMIBIE, ${ }^{5}$ den OCEAN PAPOUINDIEN ${ }^{6}$ oder die CASPERTZIENNE ANTILLAISE, ${ }^{7}$ den Bereich des PACIFIC SANDWICH ${ }^{8}$ oder die MONGOLIE TROPICALE. ${ }^{9}$ Mit Butors unverwechselbarem Humor werden die unterschiedlichsten Bereiche unseres Planeten miteinander zu Neologismen gekreuzt. Keine Area kann für sich alleine bestehen. Doch wir haben es nicht mit einer planetarischen Idylle zu tun. In den ANDES AFRONIPPONES ${ }^{10}$ vernehmen wir Signale jener Warnungen, welche die Erde zu erschüttern drohen:

Von einem Horizont zum anderen erklingen im Wechselspiel die Hörner, um vor der unmittelbar bevorstehenden Gefahr zu warnen. Sollte dies der angekündigte Kataklysmus sein? Die gesamte Provinz ist bedroht, die ganze Nation, ja der Kontinent. Allein einige Inselchen der Feuchtigkeit widerstehen. ${ }^{11}$

Die Warnungen fallen deutlich aus! Es wäre also falsch, die von Butor geschaffenen planetarischen Landschaften als den Tummelplatz harmloser Bewegungen, als den Kreuzungspunkt eines alles miteinander verbindenden arglosen Spiels zu sehen: Die Bewegungen in Butors Welt kennen die Katastrophen, die Kataklysmen. Sie erscheinen nicht nur im Zeichen ihrer natürlichen Schönheit, sondern

\footnotetext{
4 Butor, Michel: Paysages planétaires, S. 740.

5 Ebda., S. 746.

6 Ebda., S. 747.

7 Ebda., S. 748.

8 Ebda., S. 750.

9 Ebda., S. 751.

10 Ebda., S. 759.

11 Ebda.
} 
auch in dem eines drohenden Untergangs, da alles mit allem über Landschaften zusammenhängt, die planetarischen Zuschnitts sind: In denen das Lokale, Regionale oder Nationale stets das Transareale und Planetarische aufruft. So wie es falsch wäre, Butor angesichts etwa seines Engagements gegen den Algerienkrieg als unpolitischen Schriftsteller zu bezeichnen, der sich allein dem Glasperlenspiel der Literatur gewidmet hätte, so wäre es auch irrig, den französischen Autor einer Blindheit gegenüber den Klimakatastrophen unserer Tage zu bezichtigen. Er baut in seine planetarischen Landschaften die drohenden Gefahren, ja den drohenden Weltuntergang als Horrorbild ein. Denn das Reich der Raben und das Reich der Kolibris könnten eines Tages kollabieren. Die klimatischen Veränderungen sparen keinen Winkel der Erdoberfläche aus; und es sind immer wieder Erdbeben und andere Naturkatastrophen, welche die Erdoberfläche verändern, Gebirge einstürzen und Untiefen sich erheben lassen, Kanäle zwischen den Kontinenten graben und die Kontinente driften lassen, Inseln von den Kontinenten abtrennen und durch Fluten neue Becken schaffen. Die Erde ist in ständiger Bewegung und alles mit allem verbunden. Die planetarischen Landschaften Butors sind durch ihre Vielverbundenheit, ihre Multirelationalität zutiefst geprägt. Seine planetarischen Landschaften - und ich werde darauf zurückkommen - sind Archipel-Landschaften, welche die Welt weltumspannend in eine Abfolge miteinander vernetzter und diskontinuierlich zusammenhängender Inseln und Inselchen verwandeln.

Doch verlassen wir hier diese planetarischen Landschaften des Butor'schen Spätwerks und kehren wir von dieser Aussicht auf die Literaturen der absoluten Gegenwart in die sechziger Jahre und damit in eine noch andere Vorstellungswelt des französischen Schriftstellers zurück! Sein Experimentaltext Mobile aus dem Jahr 1962 ist ein Text seiner eigentlich zweiten Schaffensphase, eine Zeit, die im Grunde in die Blütezeit der französischen Theoriebildungen fällt. Nicht von ungefähr zählt Michel Butor zu den sogenannten zerebralen Autoren jener Generation, welche die Nachkriegsliteratur Frankreichs seit den fünfziger Jahren sehr stark prägte und die man auf Vorschlag eines Kritikers von Le Monde, Emile Herniot, seit 1957 (also dem Jahr von La Modification) zunehmend als Nouveau Roman bezeichnete. Butor war als promovierter Dozent ein Poeta doctus und machte nicht allein durch seine Romane, sondern auch in der Folgezeit durch eine Vielzahl hochtheoretischer, aber stets spielerischer Essays auf sich aufmerksam.

Nicht nur Roland Barthes betonte in einem wichtigen Beitrag, dass der Nouveau Roman alles andere als eine klar strukturierte, homogene und an ähnlichen Zielen ausgerichtete literarische Bewegung war. In der Tat haben sich dessen Hauptvertreter, also insbesondere Alain Robbe-Grillet, Claude Simon, Nathalie Sarraute, Robert Pinget und eben unser Michel Butor, stets und wiederholt gegen die gemeinsame Klassifizierung als „Nouveaux Romanciers“ gewehrt. Es half ihnen wenig! Wenn wir nun den Blickwinkel verändern und nicht mehr 
von der Position des Nouveau Roman her fragen, was es mit Butors Mobile auf sich hat, sondern vielmehr versuchen, sein Schreiben in den Kontext einer Literatur im Zeichen der Postmoderne zu stellen, ändert sich unsere Fragestellung. Weitaus mehr noch verändert sich unsere Interpretation und Deutung sowohl des Textes als auch von Butors Werk insgesamt.

Bei Mobile handelt es sich um einen Text, der zum einen unverkennbar als experimenteller Text angelegt ist - und damit ein gewisses avantgardistisches Verständnis auch in der Hinsicht projiziert, dass hier ein Bruch mit der literarischen Tradition herbeigeführt werden soll. Zum anderen aber geht es auch um einen Text, den man im weitesten Sinne der Reiseliteratur und der Frage des Welterlebens zurechnen könnte. Die Frage nach dem komplexen Erleben der Welt ist eine Grundfrage, die man nicht allein der Reiseliteratur, sondern auch den Literaturen der Welt insgesamt stellen muss, handelt es sich doch um eine Thematik, welche letztlich auch die Dimension der Moderne und der vielen, je nach bestimmten Areas unterschiedlichen „Modernen“ betrifft. Butors Mobile ist sicherlich kein Reisebericht im traditionellen Sinne, sondern ein hochkomplex und zugleich auch hochintellektuell angelegter Experimentaltext, der - das verschweige ich nicht - vielleicht in den Möglichkeiten, ihn zu denken und neu zu formieren, anregender ist als in den Möglichkeiten, ihn zu lesen. Freilich fordert er seine Leserinnen und Leser zu einer kreativen Lektüre auf - und eine solche wollen wir auch in Angriff nehmen!

Der Text ist sicherlich nicht jedermanns Sache und genau das will er auch ganz bestimmt - ungeachtet des etwas reißerischen Klappentextes - nicht sein. Diesen Klappentext sollten wir uns aber dennoch anschauen, sind es doch sehr oft gerade die Paratexte, welche uns den Schlüssel zu einem ansonsten nicht leicht zugänglichen Text liefern! Und schließlich ist das, was auf Vorder- und Rückseite eines Bandes abgedruckt ist, ja auch das einzige, was man bei geschlossenem Buch überhaupt lesen kann und was gerade für die Kaufentscheidung der Leserschaft von nicht zu unterschätzender Bedeutung ist. Es handelt sich um die Seite des Produkts, der Ware Buch und um dessen Zusammenhang mit der Konsumwelt, auch der Massenkonsumption. Und genau in diese Kerbe schlägt auch Michel Butors Klappentext zu seinem Band Mobile:

Atmen Sie die Luft von 50 Staaten ein!

Von Stadt zu Stadt, von Grenze zu Grenze, von der Küste des Atlantik zur Küste des Pazifik! Hunderte von Flüssen, Hunderte von Vögeln, Hunderte von Stimmen! Die Europäer, die Schwarzen, die Indianer!

Erleben Sie heute mit Ihrer Familie das Lachen, das Abenteuer, das Drama der Vergangenheit, der Gegenwart und der Zukunft Amerikas!

Reisen Sie durch einen Kontinent, durch Jahrhunderte, um den Nervenkitzel eines Spektakels zu genießen, das so groß ist wie Amerika selbst! 
Aufregung! Abenteuer! Bildung!

Vom kolonialen Neuengland bis zum Westen der Pioniere, von der mexikanischen Grenze bis zu den Häfen der Großen Seen, von Cape Canaveral bis zur Nordwestpassage!

Blättern Sie durch die Werke des großen Malers und Naturforschers John James Audubon, lesen Sie die Deklarationen von Präsident Jefferson und verfolgen Sie einen wahrhaftigen Hexenprozess!

Beobachten Sie die Amerikaner, leben Sie mit den Amerikanern, fahren Sie in ihren langen Karossen, überfliegen Sie ihre Flughäfen, entziffern Sie ihre Leuchtreklamen, flanieren Sie in ihren großen Geschäften, tauchen Sie ein in ihre endlosen Kataloge, studieren Sie ihre Prospekte, folgen Sie ihren gewundenen Straßen, schlafen Sie an ihren Stränden, träumen Sie in ihren Betten!

Mobile!

Eine Orgie an Überraschungen und Nervenkitzeln! ${ }^{12}$

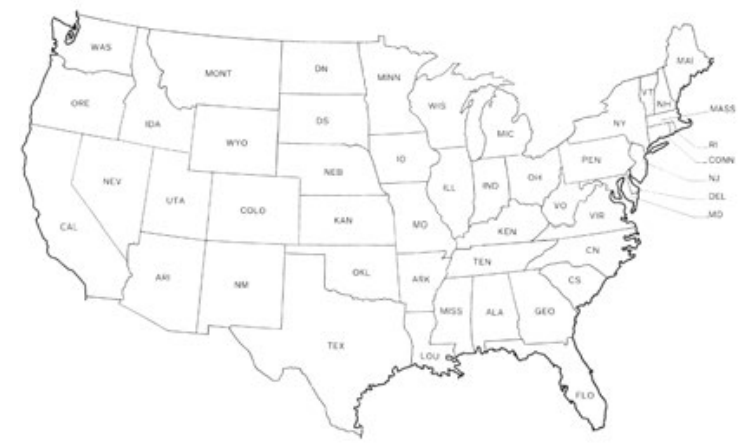

Abb. 104: Karte der USA, Abbildung aus Mobile, 1962.

Liest man die Biographie von Michel Butor, so weiß man, wie sehr er selbst von Reisen fasziniert gewesen sein muss. Schon kurz nach dem Krieg sieht man ihn auf Reisen in den unterschiedlichsten Ländern Europas, bald der ganzen Welt, wobei Aufenthalten nicht zuletzt auch an US-amerikanischen Universitäten gerade in den Jahren vor Veröffentlichung von Mobile eine große Bedeutung zukommt. Butor wusste also, wovon er sprach; und doch ist dieser Experimentaltext kein Reisebuch.

Der angeführte Textauszug zitiert durchaus das Reisefieber herbei. Er ist vergleichbar dem französischen Stil von Reisewerbung, der sich signifikant von deutschen Standardtexten dieser Art unterscheidet. ${ }^{13}$ Insofern handelt

12 Butor, Michel: Mobile: étude pour une représentation des États-Unis. Paris, Gallimard 1962, Klappentext.

13 Vgl. hierzu u. a. das Kapitel zur Werbung in Grosse, Ernst Ulrich / Lüger, Heinz-Helmut: Frankreich verstehen. Eine Einführung mit Vergleichen zu Deutschland. 4., aktualisierte und erweiterte Auflage. Darmstadt: Wissenschaftliche Buchgesellschaft 1996. 
es sich gattungsspezifisch tatsächlich um einen Werbetext, der im Übrigen zugleich eine ironische Distanz zu einer Funktion aufbaut, welche er gleichzeitig erfüllt, nämlich diejenige der Werbung für das Buch, als dessen Klappentext er fungiert. Es ist also ein Appetizer, der seine Wirkung durchaus entfalten kann, macht er doch zumindest auf den Band aufmerksam und löst bei seinem potenziellen Publikum zumindest Interesse für den marktschreierisch beworbenen Text aus.

Wir sehen an dieser Stelle den unter anderem von Roland Barthes angesprochenen unhintergehbaren Zusammenhang noch der subtilsten Literatur, die sich an eine breite Öffentlichkeit wendet, mit den Mechanismen, Strategien und Bedingungen der Massenkommunikation, gerade auch in einem ,Reisebuch', das in den Zeitraum des beginnenden Massentourismus fällt. Michel Butor wählt in seinem Klappentext die Strategie hyperbolischer Übersteigerung, die eine ironische Distanzierung erlaubt und damit in sich selbst die Problematik der Verbindung zwischen hoher Literatur einerseits und Massenkonsum wie Massenkommunikation andererseits präsentiert und integriert. Der Klappentext von Butors Mobile reflektiert dieses Spannungsverhältnis, dem die Literaturen spätestens seit den sechziger Jahren des 20. Jahrhunderts ausgesetzt sind.

Zugleich suggeriert dieser Text natürlich, dass man durch seine Lektüre selbst eine Reise unternehmen kann. Damit ist die Reisemetaphorik eingeschrieben in die Grundstruktur des Butor'schen Textes. Nun ist es eine bekannte Tatsache, dass sich die Metaphorik der Reise von Beginn der Romangeschichte an, also der Geschichte der Gattung des Romans, häufig auffinden und in frühen Romantexten nachweisen lässt. Sie nimmt eine zentrale Stellung ebenso in der Struktur von Miguel de Cervantes' Don Quijote wie in Marcel Prousts A la recherche du temps perdu, im Pícaroroman des Lazarillo de Tormes und im vorgelagerten Abenteuerroman sowie im Roman de Geste ein, bis hin zu den aktuellsten Thrillern unserer Tage in der Epoche der Massenkultur. Natürlich beschränkt sich die Reisemetaphorik als narrative und diskursive Grundstruktur nicht auf die Gattung Roman, findet sich in anderen Texten - denken Sie an die Commedia, die Göttliche Komödie Dantes, die mit dem Verweis auf einen Weg einsetzt! - und Textsorten bis hin zum Selbstverständnis und zur Selbstversicherung unseres Lebens. Denn unser eigenes Leben kann - nicht nur in der christlichen Tradition - als Reise, und sei es als Pilgerreise, angesehen werden.

Sicherlich ist im übertragenen Sinne jede Textlektüre eine Reise: von den ersten Buchstaben auf dem Umschlag bis zum Ende der Schrift und des Buches, solange wir eine lineare Lektüre pflegen. Die Reisestruktur lässt sich damit als anthropologische Konstante verstehen, ist zugleich aber eingeschrieben in die Romangattung, von unterschiedlichsten Formen des Reiseberichtes einmal abgesehen. Was sich uns aber in Butors Mobile ankündigt, ist eine Reise der beson- 
deren Art, eine Reise durch verschiedene Regionen eines Kontinents, durch verschiedene Zeiten und die verschiedenen Ethnien, welche diesen Kontinent bevölkern. Vor allem aber ist es eine Reise durch verschiedene Daseinsformen, die sich in jener Welt der Vereinigten Staaten von Amerika ein Stelldichein gegeben haben.

Damit stellt sich uns eine weitere Frage: Denn diese Welt der USA ist zugleich der geographische Raum eines Mythos der Moderne, wie er sich insbesondere seit der unmittelbaren Nachkriegszeit in nahezu allen Ländern Europas verbreitete. Selbstverständlich werden auch die alten Mythen aufgefahren, nicht zuletzt die stets von den Europäern gesuchte, aber niemals gefundene Nordwestpassage, auf welche bereits im Klappentext augenzwinkernd verwiesen wird. Im Vordergrund steht jedoch der allgegenwärtige neue Mythos des großen, modernen, vorbildhaften und zukunftsträchtigen Amerika alias USA. Michel Butor hat in all seinen Texten seit den fünfziger Jahren stets Mythen und bestimmte Mythologien eingearbeitet. In Mobile nun greift er einen modernen Mythos und mehr noch den Mythos der Moderne auf, der sich in den USA verkörpert oder den man zum damaligen Zeitpunkt von Europa aus in den Vereinigten Staaten verkörpert sah.

Einige Elemente dieses US-amerikanischen Mythos sehen Sie dabei schon in diesem kurzen Werbetext repräsentiert, etwa die Rede von einem ganzen Kontinent (der dieses Land, auch wenn wir die Vereinigten Staaten aus historischen Gründen als „Amerika“ und seine Bewohner als „Amerikaner“ bezeichnen) sicherlich nicht ist. Oder der Mythos der verschiedenen, Rassen“ und Ethnien, die sich dort ein friedliches Stelldichein im Melting Pot geben (ein nicht weniger irreführender Mythos, dem sich in unseren Tagen die Bewegung „Black Lives Matter“ entgegenstellt); oder der Mythos einer großen und großartigen Natur, die sich dort ausgebildet hätte (wozu bereits Alexander von Humboldt das Nötige sagte); oder der Mythos schlicht der Unendlichkeit, des großen Spektakels, das den europäischen Besuchern wohlige Schauer über den Rücken rieseln lasse. Hinter all diesen Mythen-Aufzählungen können wir fraglos die lächelnde Gestalt von Michel Butor vermuten.

Wir merken so beim zweiten Hinsehen, dass dieser Text aufgeladen ist nicht nur mit Formeln und Floskeln der Werbesprache, sondern auch einer Vielzahl von Amerika-Mythen, die im Europa der Nachkriegszeit hinsichtlich der USA im Umlauf waren. Auch die Anrufung, die Apostrophierung einzelner Bereiche wie etwa des Spektakels, der Erregung, des Abenteuers oder der Welt der Bildung werden in diesem Kontext letztlich zu Repräsentationsformen des Mythos oder, wie wir mit Roland Barthes sagen könnten, der bürgerlichen Mythologies, denen man im Sinne des Mythenkritikers nur mit einer Analyse von „le mythe, aujourd'hui“ beikommen kann. Ganz nebenbei sei bemerkt, dass Barthes sein 
erstes großes Erfolgsbuch Mythologies 1957, wenige Jahre zuvor, vorgelegt hatte und es zum damaligen Zeitpunkt in aller Munde war.

Wir können es uns an dieser Stelle unserer Vorlesung aus Zeitgründen leider nicht erlauben, nun auf die Mythologies von Roland Barthes zurückzugreifen und mit deren Mechanismus die verschiedenen in Butors Text integrierten Mythen zu analysieren. Ich darf Sie hier auf meinen kleinen Band Landschaften der Theorie verweisen, in welchem den Mythologies breiter Raum gegeben wird. ${ }^{14}$ Doch wichtiger scheint mir eine zweite Überlegung.

Es handelt sich ja, wie wir sahen, schon von Beginn des Klappentextes an um einen Reisetext besonderer Art. Auch der Titel verweist, freilich hochgradig polysem, auf den Status eines Bewegungstextes. Gewiss ist der Titel Mobile sehr vieldeutig zu interpretieren. Das Titellexem meint zum einen die ständige Bewegung, welche im Übrigen auch Butors früheren Texten immer eigen war, beruhten sie doch stets auf einem mobilen, vom Lesepublikum erst auszufüllenden Grundschema. Es kommt aber auch die schlichte Tatsache hinzu, dass Mobile eine Stadt in den USA ist, damit auch einen Ort innerhalb der hier dargestellten Vereinigten Staaten bezeichnet. Weiterhin schließlich - wie Sie wissen - hält Sie zum damaligen Zeitpunkt ein Erdölkonzern und eine Tankstellenkette dieses Namens gleichsam mobil und in Bewegung. Und schließlich ist Mobile natürlich auch die Bezeichnung für ein Mobile, ein ständig in Bewegung befindliches kunsthandwerkliches oder künstlerisches Artefakt, das sich in seinen jeweiligen Einzelteilen stets in Hinblick auf die wechselseitigen beweglichen Teile und deren Verhältnis zu den Betrachter*innen in beständiger Veränderung befindet.

Gerade den jungen Michel Butor haben schon sehr früh die Mobiles von Alexander Calder beeindruckt: Mobiles, die mit ihren beständigen Veränderungen ja bekanntlich nicht nur die großen Museen dieser Welt bereichern, sondern auch eine ungezählte Menge öffentlicher Gebäude und Schulen in Frankreich zieren, welche eine Vorliebe entwickelt zu haben schienen für diese Art der Kunst am Bau. Mobiles scheinen heute ein wenig aus der Mode gekommen zu sein, sind aber immer noch präsent und harren vielleicht einer baldigen Wiederauferstehung. Calders verrostete Mobiles könnten ein Lied davon singen ...

Der zweite Teil des Titels, mithin der Untertitel, ist ein wenig trockener: Studie für eine Darstellung der Vereinigten Staaten. Die Gattungsbezeichnung „Studie“ lässt Schlimmes erahnen (und zum Teil trifft es ja auch zu): dass es nämlich in diesem Text um eine große Ansammlung von Informationen und Materialien geht, welche sich auf den Gegenstand „Etats-Unis“ beziehen, die in dieser Formu-

14 Vgl. Ette, Ottmar: Roland Barthes. Landschaften der Theorie. Konstanz: Konstanz University Press 2013. 
lierung in ihrer ganzen Pluralität daherkommen. Es bleibt festzuhalten, dass die architextuelle, das heißt gattungsmäßige Selbstzurechnung, die der Text seinen Leser^innen offeriert, ein wenig wissenschaftlich anmutet.

Bereits unmittelbar nach der Widmung finden wir auf den ersten beiden Seiten des eigentlichen Textes eine Darstellung der Vereinigten Staaten. Die Doppelseite nimmt graphisch vorweg, was später grammatextuell im weiteren Verlauf des Textes ,ausgefaltet“ wird, nämlich die Art der Benutzung der immerhin 539 Seiten des Bandes, der im Querformat von oben nach unten gelesen werden muss. Die erste Repräsentation der Vereinigten Staaten ist schlicht die politisch-geographische, insofern keine Gebiete außerhalb der USA - auch nicht Alaska, Puerto Rico oder Hawaii - dargestellt werden und auch innerhalb des Territoriums der USA nur die politischen Grenzen erscheinen. Jeder Staat ist dabei durch die ihm offiziell entsprechende Abkürzung in Großbuchstaben gekennzeichnet und identifiziert, so dass diese Karte zugleich für den weiteren Text als Orientierungshilfe dienen kann.

Werfen wir nun vergleichend einen Blick auf traditionelle Reiseliteratur, so beschäftigen wir uns mit einer vielfältig verzweigten Gattung, die über einen langen Zeitraum im Schatten der Literaturwissenschaft stand und trotz ihrer ungeheuren Verbreitung und jahrhundertealten Tradition kaum einmal wissenschaftliche Beachtung fand - es sei denn, um reiseliterarische Texte dokumentarisch zu lesen. Schauen wir uns dabei die Struktur dieser Texte einmal näher an, so sehen wir rasch, dass sie im Grunde einem autobiographischen Schema in aller Regel treu sind, insoweit sie eine Ich-Figur als Protagonisten kennen. Dieses Ich zerfällt dabei - um es etwas verkürzt auszudrücken - in eine Ebene der erzählten Zeit und eine der Erzählzeit, so dass wir ein erinnertes Ich und ein erinnerndes Ich oder, wenn Sie das lieber wollen, ein reisendes Ich und ein diese Reise erfassendes, darüber berichtendes, die Reise darstellendes Ich vor uns haben. Das alles können Sie in Hinblick auf Michel Butors Mobile aber getrost vergessen!

Im traditionellen Reisebericht können Sie eine Reihe von Grundbewegungen unterscheiden, die zugleich auch von hermeneutischer Bedeutung sind, das heißt Bewegungen darstellen, die der Leserschaft gleichsam im Raum entfaltete Verstehens-Modelle darbieten. ${ }^{15}$ Das vielleicht gängigste Grundschema ist sicherlich die Kreisstruktur, wobei der Reisende von einem Punkt aus aufbricht und $\mathrm{zu}$ ihm am Ende der Reise wieder zurückkehrt. Durch diese Bewegung hat er oder sie freilich einen Erfahrungsschatz hinzugewonnen, so dass die oder der Reisende eben nicht mehr als der- oder dieselbe zu diesem Ausgangspunkt zurückkehrt.

15 Vgl. Ette, Ottmar: ReiseSchreiben. Potsdamer Vorlesungen zur Reiseliteratur. Berlin - Boston: Walter de Gruyter 2020, S. 194-235. 
Bisweilen setzen die Reisenden diesen Ausgangspunkt nach Vollzug der Kreisbewegung auch auf andere Weise in Szene, um zu dokumentieren, dass er sich auf Grund der geleisteten Verstehens-Prozesse für sie anders oder in anderem Lichte darstellt.

Ein zweites Grundmuster ist die lineare Bewegung von einem Punkt A zu einem Punkt B, wie wir es etwa bei der Pilgerfahrt im christlichen Bereich nach Jerusalem, Rom oder Santiago de Compostela, im muslimischen Bereich zu den heiligen Stätten des Islam vor uns haben. Aber natürlich ist auch eine Reise nach Memphis vorstellbar, um zu Elvis Presley zu pilgern - was auch immer Sie bevorzugen! Neben der Kreis- oder der linearen Bewegung gibt es aber auch andere Grundfiguren hermeneutischer Bewegung, so etwa eine sternförmig von einem Zentrum ausgehende Reisebewegung, die ebenfalls in bestimmten Subgattungen des (literarischen) Reiseberichts von großer Bedeutung ist.

Wir könnten dies noch wesentlich diversifizieren, und es wäre in der Tat möglich, noch weitere Formen und Unterformen hermeneutischer Bewegung in Texten der Reiseliteratur angelegt zu finden und als Verstehens-Figuren herauszuarbeiten. All dies ist gut und schön, trifft aber für den ,Reisebericht‘ unseres Michel Butor nicht zu! Denn wir finden dort nicht nur kein konsistentes Subjekt in Form eines reisenden Protagonisten, ja nicht einmal unbedingt ein Auseinanderfallen in zumindest zwei Erzählebenen oder zwei Erzählzeiten und auch keines der durchgängigen hermeneutischen Bewegungsfiguren im Text vor. Ja mehr noch: Alle kontinuierlichen Bewegungen oder Bewegungsfiguren, im Übrigen auch einer narrativen und diskursiven Kontinuität, sind absichtsvoll gebrochen, wenn auch nicht gänzlich verschwunden. Der Text charakterisiert sich durch ein ständiges Hin- und Herspringen.

Wir können diese oszillierende, springende oder hüpfende Bewegungsfigur im Grunde auf der eingangs erwähnten politisch-geographischen Landkarte der USA in etwa nachzeichnen, auch wenn dabei dann am Schluss ein Bewegungsbild entstünde, das wesentlich inkohärenter aussähe als die Darstellung aller inneramerikanischen Fluglinien sämtlicher inneramerikanischen Fluggesellschaften. Es zeigt sich zwar, dass Butors Text bisweilen einer gewissen alphabetischen Logik folgt, insofern die US-Staaten meist in alphabetischer Reihenfolge abgehandelt werden; doch ist dieses Schema gerade gut genug, um ständig hintergangen oder mit Hilfe anderer Reisebewegungen subvertiert zu werden.

Völlig unstete und vor allem diskontinuierliche Bewegungen sind dem Text zutiefst eingeschrieben. Das ständige Hin- und Herpendeln auf der nordamerikanischen Landkarte lässt keine politisch, ideologisch, geographisch, diskursiv, narrativ oder wie auch immer geartete Logik zu Stande kommen: Der Text ist auf dieser Ebene vielmehr eine Abrechnung und ein Abschied von all diesen durchgängigen Logiken. Insoweit inszeniert er zweifellos einen Bruch mit der 
traditionellen Gattung des Reiseberichts und wäre daher eher avantgardistischer als postmoderner Ästhetik zuzuordnen. Doch wir können andere Textelemente aufspüren, die eher für eine sanfte Abkehr von traditionellen Schreibformen und eine „sanfte Apokalypse“ sprechen.

$\mathrm{Zu}$ den typischen Elementen des Reiseberichts gehört zweifellos der Hinweis auf Fortbewegungsart und Verwendung unterschiedlicher Fortbewegungsmittel. In der Tat finden wir in Mobile eine ganze Reihe von Verkehrsmitteln, namentlich aber - was die Fortbewegungsarten im Raum angeht - Flugzeug und Automobil, von dem aus immer wieder eine Suche nach verschiedenen Tankstellen gestartet wird, egal ob Texaco, BP oder eben Mobile. Es handelt sich folglich vorherrschend um Verkehrsmittel, die wir der Moderne zuschreiben dürfen.

Denn Mobile besitzt auf anderen Textebenen durchaus bestimmte Formen einer recht konsequent durchgehaltenen Logik. Eine dieser Logiken betrifft die „mise en page“, also die Situierung des Textes auf der Buchseite selbst. Dabei fällt, wie bereits erwähnt, von Beginn an auf, dass nicht die einzelne Seite, sondern eine Doppelseite, also das aufgeschlagene Buch, um neunzig Grad gedreht, die Grundmatrix dieses Textes bildet. Damit wird das Querformat gewählt, so dass die Leserschaft im Grunde das Buch in anderer Leserichtung oder - wenn Sie lieber wollen - Bewegungsrichtung halten muss. In jedem Fall wird eine traditionelle Lesart unmöglich gemacht, auch wenn bestimmte paratextuelle Elemente in herkömmlicher Form gedruckt sind.

Denn die veränderte Leserichtung betrifft nur den ,eigentlichen` Text des Buches: Weder Titel noch Klappentext, weder Titelseite noch Karte oder die Hinweise auf andere Werke dieses Autors sind im Querformat gedruckt, sondern dürfen oder müssen in gewohnter Leserichtung gelesen werden. Dies bedeutet, dass sich der eigentliche Text von seinen Paratexten sehr stark absetzt und - was mir nicht unwichtig erscheint - auch grafisch von ihnen deutlich unterschieden ist.

So ließe sich durchaus sagen, dass die Struktur von Mobile der eines Mobile ähnlich ist, bei dem die Rahmenstruktur in gewisser Weise oft fest montiert sein muss, gerade um eine beständige Bewegung der einzelnen beweglichen Teile zu gewährleisten. Diese mobilen Teile sind auf der Seite wiederum grafisch voneinander abgesetzt: So wurden zum Beispiel alle Städtenamen und Namen von Bundesstaaten, die sie ergänzen, in Majuskel gesetzt, wobei gerade auch bei Repetitionen eine gewisse grafische Grundstruktur auf der Seite erzeugt wird. Daneben gibt es als Grundformen des grammatextuellen Aufbaus Antiqua-Schrift und Kursivierung, welche als zusätzliche strukturierende Textelemente eingesetzt werden.

Die Paginierung erfolgt jeweils über die rechte, also die ungerade Buchseite, was keineswegs beiläufig oder gar belanglos ist, da hierdurch angezeigt wird, dass 
es sich bei einer Doppelseite jeweils um eine Einheit handelt. Gleichwohl werden die Doppelseiten als zwei Seiten gezählt. Dabei entstehen Seiten-Bilder, die in der Tat etwas von der „mise en page“ konkreter Poesie und vielleicht mehr noch des Bildgedichts avantgardistischer Herkunft transportieren. Zugleich werden die vielen Brüche und Diskontinuitäten innerhalb des Textes sichtbar gemacht, zeigt der Text doch in seiner Gänze eine grundlegende Patchwork-Struktur grammatextuell an.

Der ,eigentliche‘, fortlaufende Text von Butors Mobile beginnt mit der Erwähnung der Stadt Cordoue in Alabamas tiefem Süden und setzt sich - auf der nächsten Doppelseite - im Norden mit Cordue/Alaska fort. Damit wird die bereits ins Spiel gebrachte alphabetische Logik erstmals aufgerufen, zugleich aber auch die Reisebewegung in eine vom sprachlichen Material vorgegebene Anordnung gebracht. In der Tat ist es dieses Material, das für Butor stets als solches welthaltig ist: Es verleiht dem gesamten Text die Grundstruktur.

Dabei werden häufig Zitate aus den verschiedensten Bezugstexten angegeben und einbezogen, so dass Mobile von Anfang an signalisiert, dass es im Text nicht nur einen einzigen Autor, eine einzige Erzählerfigur gibt. Dieser experimentelle Text erweist sich bereits auf dieser Ebene als mobil, ganz abgesehen davon, dass die Identität der Städtenamen kombiniert mit der Differenz der BundesstaatenBezeichnungen ein Spiel von Identität und Differenz in Gang setzt. Dieses ist ebenfalls nicht an der Realität dieser Städte und Staaten, also nicht an ihrer außersprachlichen Referentialität ausgerichtet, sondern an der sprachlichen Dimension oder - gerade was wir in Hinblick auf die Wichtigkeit von Klangeffekten wahrnehmen können - an der poetischen Funktion der Sprache im Sinne Roman Jakobsons. Nicht die referentielle, sondern die poetische Funktion dominiert in diesem gattungsmäßig sehr vielfältigen, mit verschiedensten literarischen Genres vom Reisebericht bis hin zur konkreten Poesie spielenden Mobile.

Das Spiel von Identität und Differenz wird selbstverständlich noch auf anderer Ebene wichtig: derjenigen immer neuer Kontexte für die immer wieder wiederholten Strukturen oder Textelemente. So wird das wiederholt auftauchende Selbe durch einen anderen Kontext in etwas Anderes und Weiteres verwandelt, ohne doch aufzuhören, zugleich das Selbe und das Andere zu sein. ${ }^{16}$ Dies ist eine Frage der Serienbildung und „Iterabilität“, die gerade für die Philosophie Jacques Derridas und insbesondere sein Theorem der „différance“ von Bedeutung ist.

Nehmen wir uns nun aber eine erste Doppelseite von Mobile genauer vor:

16 Vgl. Descombes, Vincent: Das Selbe und das Andere. Fünfundvierzig Jahre Philosophie in Frankreich 1933-1978. Aus dem Französischen von Ulrich Raulff. Frankfurt am Main: Suhrkamp 1981. 
MARSHALL, sur la baie de Tomales, comté de Marin ou comté Marin, CALIFORNIE, l'État le plus peuplé après le New York, à la frontière de la province mexicaine de California Baja, - la réserve d'Indiens de Manzanita.

Fe rêvais de San Francisco.

Esso, - sur les montagnes l'achillée d'Occident, dont les Indiens se servaient pour composer leurs médecines; sur les plateaux, les gaillardes dites "roues de feu "; dans les déserts, le pavot blanchâtre épineux.

BENTON, entre la forêt nationale d'Inyo et celle de Toyabe, près du lac Noir, dans la Sierra Nevada, dans l'immense et quasi désert comté de Mono, État du séquoia, - la réserve d'Indiens Inajas.

L'avion dans lequel je voyageais vers San Francisco s'est arrêté trois fois à Los Angeles : à Long Beach, à l'aérodrome international, à Burbanks. fe voyais défiler sous mes yeux les hectares et hectares de petites rues perpendiculaires faiblement éclairées...

Dans les forêts du Nord, les trilliums du Pacifique, pétales blancs et feuilles vertes trois par trois, et la fleur jumelle avec ses clochettes roses par paires.

GREENWOOD, comté d'El Dorado.

Ge suis arrivé la nuit à San Francisco. Il y avait peu de lumières sur la baie. Mais le matin...

fe rêve de San Francisco.

Le palmier de Washington, dont les palmes épineuses, en séchant, recouvrent le tronc d'un manchon de rude fourrure ocre, - quand il est cinq heures du matin à

CONCORD, près de l'embouchure du fleuve Sacramento dans la baie de San Pablo, qui donne elle-même dans la baie de San Francisco,

Abb. 105a: Michel Butor: Mobile, 1962, S. 25.

Wir haben es an dieser Stelle mit dem Übergang zum Staat Kalifornien zu tun, zu dem - wie häufig innerhalb dieses Textes - zunächst eine quantitative Angabe gemacht wird, welche ihn sehr bewusst mit dem Staat New York - und vermittelt mit allen anderen Staaten des Bundesgebietes - in Verbindung setzt. Diese relationale beziehungsweise multirelationale Struktur, die innerhalb von 
Mobile eine essentielle Funktion erfüllt, wird ergänzt durch die Erwähnung von Begrenzungen, zunächst der Außengrenzen räumlicher Natur zu Mexiko, das bekanntlich über eine Provinz namens Niederkalifornien verfügt. Sobald die Leserschaft weiß, dass dieses Territorium nicht umsonst einen spanischen Namen orientalisierenden Inhalts trägt, versteht sie zugleich, dass die Bildung des Staates Kalifornien mit einer historischen Ausgrenzung zusammenhängt. Das zentrale Thema der Grenze scheint dabei mehrfach auf. Am Ende des Satzes erfolgt ein Verweis auf die Manzanita-Indianer und deren Reservat im Staat, womit eine exkludierende Grenzziehung eingespielt wird, die innerhalb Kaliforniens erfolgt und im Grunde eine Art innere Grenze und mehr noch (rassistische) Ausgrenzung versinnbildlicht. Die Erwähnung der indigenen Bevölkerung, die in Reservaten zusammengepfercht lebt, erfolgt ganz bewusst im Text, macht sie doch auf das aufmerksam, was bis heute in den Vereinigten Staaten von Amerika unterdrückt wird. In jedem Falle werden wesentliche Elemente der Geschichte Kaliforniens und der USA aufgerufen, ohne dass dies explizit gemacht würde.

Nach Erläuterungen in Antiqua-Schrift findet sich jeweils ein Satz in der ersten Person Singular. Es handelt sich um einen einfachen Aussagesatz in Kursivschrift, der vom Traum dieses Ichs von San Francisco berichtet oder zeugt. Das „Ich träumte von San Francisco“verweist auf den amerikanischen Traum, den Zug nach Westen, die Mythologie des amerikanischen Westens und zugleich die mit Gold und Reichtum besetzte Mythologie der USA insgesamt. Danach folgen die Erwähnung einer Tankstelle beziehungsweise eines Ölkonzerns und der Folgen der von diesem Treibstoff ausgelösten oder ermöglichten Bewegungen. So wird die Wahrnehmung von Landschaftselementen projiziert, in welche freilich nicht allein die Natur, sondern die Geschichte eingeschrieben ist. Die Faszinationskraft der Wüste, die nicht nur in dieser Passage hervortritt, hat uns bereits in der Vorlesung zur Reiseliteratur hinsichtlich Jean Baudrillards Darstellung der USA unter dem Titel Amérique beschäftigt. ${ }^{17}$ Aber zurück zu Mobile!

Der folgende Abschnitt in Kursivschrift berichtet von einer Reise im Flugzeug nach San Francisco, in einem Flieger, der paradoxerweise dreimal in Los Angeles auf verschiedenen Flughäfen zwischenlandete, was gewiss die Größe dieser Riesenstadt verdeutlicht. Neben die Fortbewegungsart des mit dem Mythos USA verbundenen Automobils tritt nun jene des Flugzeugs, das die Größe und Weite des Kontinents assoziiert. Das Ich sieht unzählige Straßen unter sich hinwegziehen, eine gewisse Unendlichkeit urbaner und infrastruktureller Anlagen, die durch drei abschließende Pünktchen grafisch versinnbildlicht werden. Sie erinnern an

17 Vgl. Ette, Ottmar: ReiseSchreiben, S. 609-627. 
ein avantgardistisches Gedicht, was uns darauf aufmerksam machen mag, dass Michel Butor in seinen Anfängen derartige Gedichte verfasste.

Dann erfolgen wiederum in Antiqua-Schrift Hinweise zur Vegetation Nordkaliforniens, die wie die zahlreichen ornithologischen, einem Standardwerk Audubons entnommenen Angaben oft in den fortlaufenden Text eingestreut sind. Die nachfolgenden Namen rufen geradezu mythische Bestände auf, zunächst „Greenwood“, was unmittelbar an die Baumvegetation anschließt, dann die Bezeichnung „El Dorado“, die auf einen herausragenden, die europäischen Eroberer faszinierenden Mythos ganz Amerikas verweist, der in die Identität nicht nur des heutigen Kolumbien als Projektionsfläche von Utopien und Phantasien tief ins kollektive Gedächtnis eingegraben ist. Der Goldrausch in Kalifornien bildet gleichsam eine Wiederholungsszene von Träumen der ersten Konquistadoren, die diesem westlichen Bundesstaat einst seinen Namen gaben, lange bevor er von den USA nach einem unrechtmäßigen Krieg in Besitz genommen wurde.

Danach wird der kursiv gesetzte Ich-Erzählerdiskurs wieder aufgenommen: Eine gewisse Fortsetzung tritt ein, insoweit nun nachts das Ich in San Francisco ankommt. Statt aber zu einer narrativen Entwicklung überzuleiten und auf diese Weise eine lineare Erzählstruktur einzuführen, welche die des Reiseberichtes wäre, enttäuscht der Text die Lesererwartung und kehrt wieder an seinen Ausgangspunkt zurück. Freilich ist nun das „Imparfait“ in ein Präsens verwandelt, eine Änderung, die auf der Zeitachse eine grundsätzliche Bewegung aus der Vergangenheit in die Gegenwart nahelegt. Die nachfolgende Erläuterung zur Vegetation wird plötzlich durch einen Gedankenstrich und eine Zeitangabe unterbrochen. Diskontinuierlich wird ein Concord in Kalifornien in Bezug gesetzt zu einem absichtsvoll auf der nächsten Seite befindlichen Concord in einem anderen Bundesstaat, so dass die Ungleichzeitigkeit der Zeit in den USA durch den Verweis auf die verschiedenen Zeitzonen eingeblendet werden kann. Michel Butors Mobile verweigert sich beharrlich allen kontinuierlichen Erzählformen.

Anhand dieses Textauszuges sollte deutlich geworden sein, dass allein auf dieser Doppelseite eine ganze Reihe verschiedener Textelemente miteinander kombiniert wurden: die Angaben von Namen der Städte und Staaten, Hinweise zu Vegetation, zum Teil auch zu Landschaften und deren Nutzung, Texte zur Natur im Allgemeinen. Unterbrochen werden sie von Fragmenten eines Diskurses des Reisenden, der sich mit Hilfe zweier Verkehrsmittel, mit Auto und Flugzeug, fortbewegt, wobei beide Fortbewegungsweisen miteinander verbunden werden. Doch beziehen wir die unmittelbar nachfolgende Doppelseite in unsere Deutung mit ein: 


\section{BIENVENUE EN CAROLINE DU NORD}

il fait déjà jour depuis longtemps à

CONCORD, temps oriental, où vous pourrez demander, dans le restaurant Howard Johnson, s'ils ont de la glace à l'abricot.

$\begin{array}{ll}\text { La mer, } & \text { les vagues, } \\ \text { le sel, } & \text { le sable, } \\ \text { l'écume, } & \text { les algues. }\end{array}$

Les Indiens Cherokees invitèrent les missionnaires à venir s'installer parmi eux et à ouvrir des écoles pour leur enseigner leurs secrets; mais ceux-ci, jugeant que la langue des Indiens ne pouvait pas s'écrire, et ne modifiant nullement les méthodes qu'ils avaient apportées d'Angleterre, n'obtenaient que peu de résultats...

Noir.

Le marais d'Angola, "Hello, Al! „ - Passée la frontière du Sud-Ouest,

CONCORD, GEORGIE, côte atlantique (for whites only) (dans les États du Sud, une partie des autocars ou des tramways est interdite aux gens de couleur).

La mer,

$\begin{array}{ll}\text { la houle, } & \text { la marée, } \\ \text { les iles, } & \text { la brise, } \\ \text { Houx noirs, } & \text { les lagunes. } \\ & \text { lauriers-roses, myrtes dahoon. }\end{array}$

Les tumulus d'Ocmulgee révèlent les traces de six civilisations successives, la plus ancienne pouvant remonter jusqu'à 8000 avant fésus-Christ, la plus récente mourant au XVIII siècle...

Noire.

Mobile 27

Abb. 105b: Michel Butor: Mobile, 1962, S. 27.

Sie ist überschrieben mit einem Willkommensgruß in North Carolina, von welchem der auf der vorigen Doppelseite begonnene Satz unbeeindruckt abgeschlossen wird. Das Umblättern der Seite repräsentiert den Wechsel der Zeitzone, allerdings nicht vorwärts in der Zeit, sondern vielmehr zurück. $\mathrm{Zu}$ den ständig wiederkehrenden Textelementen zählt die Frage, ob in einem immer wieder anderen Restaurant bestimmte Eissorten vorhanden sind. Der 
sich daran anschließende Kursiv-Text besitzt einen ausgeprägt lyrischen Charakter: Er ist in seiner Anordnung dreimal zweigeteilt, wobei auf der linken Seite die Elemente des Meeres beziehungsweise Wassers stehen (Meer, Salz und Schaum), während auf der rechten Seite mit Wellen, Sand und Algen gleichsam ergänzende Elemente aufgeführt werden. In der Treppenartigen Anordnung dieses lyrischen Intermezzo wird deutlich die Bewegungsstruktur des Einschubs erkennbar.

Im unmittelbaren Anschluss folgt ein Text in Kursivschrift, der wiederum linear-diskursiv angeordnet ist und von den Cherokee-Indianern berichtet. Dabei handelt es sich um eine nicht stattgefundene Kommunikation, zu welcher die Cherokee im Grunde bereit waren, nicht aber die aus England stammenden Missionare, die ihre Methoden nicht den von ihnen zu missionierenden Indigenen anpassen wollten. Hier wird also die indigene Thematik wieder aufgegriffen. Es geht um den gescheiterten Versuch eines interkulturellen Dialogs und zugleich um die Problematik der Alterität, die in jeder Form des Reiseberichts stets von Bedeutung ist. Auf dieser Ebene macht Butors Mobile keine Ausnahme: Dort verkörpern gerade die Indianer diese Alterität und mehr noch Fremdheit, welche längst in sogenannte Reservate zurückgedrängt ist. Im Übrigen wird das Lesepublikum später erfahren, dass die Sprache der Cherokee von einem Indianer verschriftlicht, also ins Alphabet überführt wurde, was die englischen Missionare noch nicht einmal versucht hatten. Im Grunde berichtet Mobile von gänzlich asymmetrischen Machtbeziehungen, die auf den zweifellos rassistisch begründeten Ausschluss weiterer Kulturen und deren letztliche Beseitigung abzielen.

Darauf erfolgt eine erneute Grenzüberschreitung entlang des Paradigmas „Concord“: diesmal ein Concord in Georgia. Erneut erfolgt der Hinweis auf eine rassistisch begründete Ausgrenzung, diesmal nicht der indigenen, sondern der schwarzen Bevölkerung hinsichtlich der Verkehrsmittel, mithin der Mittel ihrer Bewegung im Raum. Damit wird implizit der marktschreierische Klappentext mit seiner Erwähnung der unterschiedlichen Ethnien, die in den USA zusammenlebten, ad absurdum geführt beziehungsweise als beschönigender und die Machtverhältnisse ausblendender Text entlarvt. Daran schließt sich eine kursiv gesetzte Passage an, in welcher das Meer in die Vegetation übergeht, aus einem zweigleisigen Schema ausbricht und wieder in Bewegung gerät: Eine dritte Spalte wird eröffnet. Es folgen erneut in Kursivschrift Hinweise auf sechs aufeinanderfolgende Zivilisationen, erkennbar an den Tumuli der Ocmulgee-Indianer, und ein geschichtlicher Raum wird durch diese aufeinanderfolgenden indigenen Zivilisationen ausgespannt, der sich von 8000 v. Chr. bis ins 18. Jahrhundert erstreckt. Erneut wird also die Dimension der Zeit angesprochen, insoweit den indianischen Kulturen der Maßstab abendländisch-christlicher Zeit entgegen- 
gehalten oder übergestülpt wird. Dass dieser Zeitraum gerade im 18. Jahrhundert zu Ende ging, könnte auf jene große geschichtliche Bewegung zurückgehen, die sich am Ausgang des Jahrhunderts als Aufklärung und Epoche einer abendländisch bestimmten Moderne inszenierte. Diese Moderne wird in Mobile zweifellos hinterfragt.

Die Gedichtstruktur dieser verschiedenen Passagen, das sorgfältige Ineinander-Weben unterschiedlichster Diskursfetzen, Ortsnamen, historischer oder kultureller Verweise, Sprachspiele oder Gedichtformen lassen einen Text entstehen, in dem sich wie in einem Kaleidoskop die einzelnen Elemente in ihren wechselseitigen Beziehungen stets neu anordnen. Diese Grundelemente, die ständig durch die Hinzufügung neuer Elemente in Bewegung gesetzt werden, bilden die offene Strukturierung dieses literarischen oder textuellen Mobile unendlicher Sprünge und Brüche zwischen den verschiedenen Grenzen kultureller Diversität. Mobile ist ohne jede Frage ein kulturkritischer Bewegungs-Text, der nicht in der Form seines marktschreierischen Werbetextes funktioniert.

An diesem Punkt sei es erlaubt, einen kurzen Seitenblick auf einen im Jahr 1962 von Roland Barthes für die Zeitschrift Critique verfassten und in die Essais critiques aufgenommenen Text über Michel Butors Mobile zu werfen! ${ }^{18}$ Barthes betonte gleich zu Beginn seines Versuchs über Butor, dass dieser mit seinem Band die Idee des Buchs selbst verletzt habe. Barthes ging in seiner Aussage noch einen Schritt weiter, indem er ausführte, wie hier doch absichtsvoll die „idée même de la littérature“ in Frage gestellt worden sei. Die im Buch wohltätigen Metaphern seien das Gewebe, das man webt, das Wasser, das fließt, das Mehl, das man zubereitet, der Weg, dem man folgt, der Vorhang, der enthüllt und vieles mehr. Dabei handele es sich letztlich um eine Struktur, welche die eines „Bricolage“ sei, einer Bastelarbeit also, die man aus den unterschiedlichsten Materialien herstelle und verfertige.

Zugleich habe Butor die Idee verletzt, dass ein Buch gleichsam aus sich selbst entsteht, organisch sich entwickelt; vielmehr sei der Konstrukt-Gedanke entscheidend. So rücke die schriftstellerische und mehr noch textuelle Arbeit in den Vordergrund, das Gemachte: Die Arbeit werde als solche nicht verborgen. Butors Buch ergebe sich gerade nicht in die Idee des „continu“, in welches alles hineinfließen müsse. Ein „livre continu“, so Barthes etwas abfällig, müsse einer „critique cosmétique“ zugehören. Das Verbot der Kritik laute, dass man resümieren und bewerten, niemals aber in zu kleine Bestandteile aufteilen dürfe. Deshalb werde das „livre discontinu“ auch nur in ganz bestimmten Bereichen toleriert,

18 Vgl. Barthes, Roland: Littérature et discontinu. In (ders.): Essais critiques. Paris: Seuil 1964, S. 175-187. 
nämlich als fragmentarisches, unvollendetes Werk, als Aphorismus, insofern dieser als Vollform inhaltlicher Art geachtet werde.

Butors Buch verstoße auch gegen den längst mythisierten Charakter der ,Entwicklung،, eines sich entwickelnden Buches. Der generelle Plan in Mobile sei „nul“, das Detail hingegen auf die Ebene einer Struktur gehoben worden. Die Ideen würden in Mobile nicht entwickelt, sondern lediglich verteilt. Barthes betonte die Wichtigkeit der Taxonomie, wie sie nun überall in den Humanwissenschaften deutlich werde: Sag mir, wie du klassifizierst, und ich sage Dir, wer Du bist. Der Verfasser der Mythologies verteidigte in diesem Essay auch die alphabetische Anordnung in Butors Mobile, eine Anordnungsweise, die er selbst wenige Jahre später zu einer wichtigen Grundstruktur eigener Texte und insbesondere seiner experimentellen Autobiographie Roland Barthes par Roland Barthes machen sollte. Nicht umsonst nannte Barthes das Alphabet jenseits der mystischen Dimension von Alpha und Omega auch den „degré zéro de classement“. Er betonte darüber hinaus, dass es in unserer Kultur immer sehr wenig Platz für das Neutrale, das Neutrum (,le neutre“) gegeben habe, dass man im Leeren stets nur die Negativität erblickt habe. Die Darstellung der USA bei Butor sei mithin eine Art „addition d'étoiles“: Die USA seien wie etwas Enzyklopädisches erobert worden, nämlich Staat nach Staat.

Roland Barthes merkte im Übrigen an, wie bedeutungsvoll in einem Land, das unter einer permanenten Identitätskrise leide, der Mangel an Ortsbezeichnungen sei. Einem zu großen Kontinent stehe ein zu kleines Lexikon gegenüber: Ein ganzer Teil Amerikas ergebe sich aus diesem „frottement des choses et des mots“, dieser Reibung zwischen Worten und Dingen - wie er in einer prospektiv Foucault'schen Wendung hinzufügte. Es handle sich bei Mobile nicht um eine modernistische Darstellung der Vereinigten Staaten, sondern um eine Tiefendarstellung, eine tiefreichende Anamnese, die darum auch besonders bedeutungsvoll sei, weil sie von einem Franzosen stamme.

Barthes verteidigte das Buch von Butor gegen den Vorwurf, eine „pensée en miettes“, ein in Scherben zerfallenes Denken zu sein. Mobile mache das „,continu“, an das wir in unseren Lektüren gewohnt seien, durchaus bemerkbar. Er fügte dem die ironische Frage hinzu, wer es denn wagen würde, Webern oder Mondrian vorzuwerfen, sie hätten eine Kunst in Scherben produziert. Das Diskontinuierliche sei von der Kunst im Übrigen nicht erfunden worden, denn das Diskontinuierliche sei der fundamentale Status jedweder Kommunikation. Die klassische Möglichkeit einer Antwort hierauf sei die Variation gewesen, doch gebe es auch andere Möglichkeiten wie jene der Translation. Schon Claude Lévi-Strauss hatte die Vermutung angestellt, dass jede Erzählung durch die Mobilisierung rekurrierender Strukturen in Gang gesetzt werde. Damit falle die Verantwortung der Wahl auf das Werk selbst. Butor habe bewusst betont, dass er ein konstruiertes Werk gebaut 
habe, und er hob die Wichtigkeit des Modells, der „maquette“, hervor. Für Roland Barthes war die Frage Butors nach der „composibilité“ der Welt von Bedeutung. Er sprach logischerweise auch von der „Zusammensetzbarkeit“ des amerikanischen Kontinents und verwies auf die alten Vorfahren der gewaltigen Katalogstrukturen, die Butors Buch über die USA charakterisierten, wenn auch seine Vergleiche mit Homer oder Aischylos etwas ins Weite führen. Damit aber gelang es Barthes paradoxerweise am Ende seines Essays, Butor wieder in eine Traditionslinie abendländischer Kultur und Literatur zurückzuholen, eine Bewegung, die freilich die Mobilität von Barthes' eigenem Essay nicht weniger in Szene setzte als die von Butors Mobile.

Ich wollte Ihnen mit diesem kleinen Ausflug zu Roland Barthes aufzeigen, wie weit bereits zeitgenössisch ein tiefer gehendes Verständnis dieses französischen Experimentaltextes gediehen war und wie früh man das subversive Potenzial von Mobile in Frankreich bereits erkannte. Wie im Reisebericht des 18. und 19. Jahrhunderts wird in Butors Mobile auf Elemente der Natur wie Vegetation, Anbau von Früchten oder auf ornithologische Fakten aufmerksam gemacht, wobei witzigerweise auf Seite 77 der amerikanische „Butor“ erscheint, wodurch der Autorname in den fortlaufenden Mobiltext selbst eingeschrieben wird. Durchaus in der Tradition des Reiseberichts erfolgt ein erneuter Verweis auf die amerikanischen Ureinwohner, wobei der Verweis auf die Indianerreservate die Untergangsgeschichte der indigenen Bevölkerung einblendet. Freilich bildet die indigene Bevölkerung neben den Schwarzen und den amerikanischen Europäern die dritte wesentliche Gruppe, anhand derer das ethnische Diversitätserleben literarisch repräsentiert und Zusammenleben in den Vereinigten Staaten kritisch reflektiert werden kann.

Gerade auch die ältesten Kulturen wie jene der Sandia-Indianer im Südwesten der USA - eine Zivilisation, die über eine Geschichte von 25000 Jahren verfügt - finden beständige Erwähnung in Mobile. Dabei erscheinen die USA in einer Tradition, die sich seit der Entdeckungsgeschichte des 16. Jahrhunderts als roter Faden ständiger Eroberung und Erweiterung durch ihre Geschichte zieht. Durchaus in Fortsetzung europäischer Traditionslinien kommt die Frage auf, inwieweit ein friedliches und gleichberechtigtes Zusammenleben in Differenz zwischen Menschen unterschiedlicher Herkünfte, aber auch zwischen dem Menschen und den verschiedensten Dimensionen der Natur möglich ist. Wir wohnen in Mobile zwar sehr wohl der Hervorhebung unerhörter Größe und Vielfalt des Landes bei, sehen zugleich aber deutlich, wie die Vereinigten Staaten von Amerika als Mythos der Konvivenz von Menschen unterschiedlichster Herkunft, aber auch des Menschen mit der ihn umgebenden Natur dekonstruiert werden. 
Die auf den ersten Blick überraschende, ständige Erwähnung der indigenen Bevölkerung - von Beginn des ,Reisebuchs‘ an - funktioniert nach demselben Schema, das dem der Ortsnamen in ihrer Verkopplung mit den jeweiligen Staaten der Union zu Grunde liegt. Es ist das Schema von Identität (die indigene Bevölkerung) und Differenz (der jeweilige Stamm oder die jeweilige Zivilisation). Darüber hinaus ergibt sich eine weitere Dimension: nämlich die der Serie. Das serielle Muster findet sich, arbeitet man sich erst einmal im Text langsam voran, im Grunde auf allen Ebenen. Immer wieder werden verschiedene Eissorten abgefragt, wobei die konkrete Eissorte jeweils wechselt; immer wieder gibt es dieselben Begrüßungsformeln mit „Hello“, wobei jeweils die Namen wechseln; immer wieder lesen verschiedene ethnische Gruppen ihre Zeitung, wobei der Ort und auch die Sprache jeweils wechseln; immer wieder wird getankt, wobei die Erdölfirma jeweils wechselt; immer wieder wird geflogen, wobei die Flugorte und Zeiten jeweils wechseln; und immer wieder gibt es Indianer, wobei die Orte und Kulturen jeweils wechseln.

Das serielle Element ist paradoxerweise gerade jenes, das Mobilität in die Abfolge bringt. Die Repetition wird durch eine jeweils neue Kombinatorik, eine neue Konstellation in Bewegung gebracht und damit das Element der Differenz just aus der Identität der Serie heraus entfaltet. Wir werden noch sehen, dass dieses Spiel von Wiederholung und Differenz von Jacques Derrida in seiner Begriffsprägung der „différance“ auf Ebene des philosophischen Diskurses entfaltet wurde. Dabei ist es überflüssig zu betonen, dass das serielle Element in der zeitgenössischen E-Musik der Moderne ein grundlegendes Verfahren der Produktion von Musikstücken war. Der stets synästhetische Verbindungen zwischen den Künsten suchende Michel Butor wusste dies wohl.

Immer wieder werden auch Elemente einer erotischen Geschichte eingeblendet, wie etwa der „corps morcelé“ einer offensichtlich schwarzen Frau, deren Bauch zwischen einem aufgeknöpften Korsett oder den Schenkeln sichtbar wird. Im Gegensatz zu den Elementen dieser „histoire érotique“ wird die eigene Familie beschrieben, die mit auf die Reise genommen wurde. Zwischen beiden Polen ergibt sich eine vom Lesepublikum zu konstruierende Spannung, insoweit auch das „Je divorce“, das „Ich lasse mich scheiden“, von der Ich-Erzählerfigur durchdekliniert wird. Eine völlige Klarheit ist in diese Biographeme aber nicht zu bekommen: Sie ist in Mobile nicht beabsichtigt. Ein in diesem Zusammenhang für das Ich bedeutungsvolles Element ist das Meer, von dem sich das Ich Veränderung, Verbesserung und nicht zuletzt auch Heilung erhofft. Ein Element der Natur, auf das immer wieder zurückgegriffen wird, insofern es in den verschiedensten Bundesstaaten erscheint. 
Ich möchte Ihnen zum Abschluss zwei weitere Doppelseiten aus Michel Butors Mobile vorstellen, die jeweils komplementäre und unsere bisherigen Beobachtungen und Überlegungen ergänzende Aspekte beinhalten:

- Rambler,

WASHINGTON, cf. de La Fayette, OH., État de vaches.

En 1772, David Zeisberger et d'autres Frères Moraves firent bâtir des villages à des Indiens Delawares qu'ils avaient convertis. En 1782, pour éviter tout conflit avec les Européens de Pennsylvanie, les missionnaires jugèrent plus sage de leur faire quitter leurs maisons de Gnadenhütten (les Chaumières de la Grâce) et de se replier à la "Ville des Prisonniers ", aujourd'hui Upper Sandusky, Ohio. Mais comme ces missionnaires leur avaient donné mission de retourner moissonner leurs anciens champs de maïs, les Indiens furent découverts par des miliciens de Pennsylvanie, sous les ordres du colonel David Williamson, qui les désarmèrent, les ligotèrent et les exterminèrent à coups de haches et de gourdins, car ils voulaient économiser leurs munitions. 35 hommes, 37 femmes et 34 enfants furent ainsi massacrés. Ils chantèrent jusqu'à leur mort les cantiques que les missionnaires leur avaient

- Studebaker, enseignés.

"Allô, je voudrais Cambridge, Ohio ", - l'église de la Science chrétienne, - les rivières du Portage et de Sandusky qui se jettent dans le lac Érié, - la rivière du Serpent-à-Sonnettes qui rejoint la rivière Scioto, - ou une perle solitaire en pendentif, "savam-

ment cultivée, produite scientifiquement dans une huitre vivante nourrie trois ans au moins dans les chaudes eaux du Japon. Le romanesque, le charme, la flatterie des perles de culture est éternelle - elles ne perdent jamais leur beauté "

Un rayon de soleil oblique sur les feuilles mouillées.

WASHINGTON, où vous pourrez demander,
dans le restaurant Howard Johnson,
s'ils ont de la glace à la cerise.
- Thunderbird,
"... fe ne cherche rien que l'honneur de Son nom,
et que nous, qui sommes Son ouvrage, puissions faire
ce qui lui plait. L'homme qui vous apportera ceci est
mon ami très cher, sobre, sage et aimant, vous pou-
vez lui faire confiance..."

- Willys 》.

Informations :

«... Et qu'ayant peu de personnes nobles parmi eux, les étrangers bien nés doivent être grandement respectés, et bien sûrs d'obtenir aisément les meilleurs de ces offices qui feront toute leur fortune; que les gouverne-

Mobile 125

Abb. 105c: Butor, Michel: Mobile, 1962, S. 125. 
Auf dieser Seite finden Sie in der grammatextuellen Anordnung, der „mise en page“ also, innerhalb der Räumlichkeit einer doppelten Buchseite, die bei der Leserschaft längst durch Routine zur Selbstverständlichkeit geworden ist, in der linken Spalte eine Reihe berühmter nordamerikanischer Automarken, die von der vorherigen Doppelseite gleichsam herüberschwappt. Ganz nebenbei und beiläufig wird dadurch an die Urform des Schriftsupports, an die Papyrusrolle, erinnert.

Michel Butor beschäftigt sich auf dieser Seite erneut mit den großen amerikanischen Mythen. Gerade der US-amerikanische Studebaker ist zu einem Mythos des American Way of Life geworden. In Kontrast hierzu finden Sie in Kursivschrift unter dem Ortsnamen Washington, wie immer in Großbuchstaben, ein Zitat, das ein Ereignis der Jahre 1772 bis 1782 beleuchtet. Es beinhaltet das Schicksal eines indianischen Stammes, der von den Mährischen Brüdern (also einem deutschen Orden) missioniert wurde, sich unter dem Druck der vorrückenden Besiedelung in sicherere Bereiche zurückziehen musste, gleichwohl aber gegen Ende des 18. Jahrhunderts - Sie sehen, es handelte sich nicht um einen Zufall! - in das Räderwerk der US-amerikanischen Expansion geriet und im Zeichen der fortschreitenden Moderne bestialisch ermordet wurde.

Die Indianer waren in gewisser Weise schon vor ihrer Ermordung kulturell tot, sangen sie doch noch im Sterben jene Lieder, welche die Mährischen Brüder ihnen beigebracht hatten und nicht etwa die ihrer eigenen Kultur zugehörigen Totenlieder. Danach erfolgen im Text ein Zeitsprung und zugleich auch ein grafischer Sprung mit der Einblendung eines Telefongesprächs sowie eines Verweises auf verschiedene Flüsse dieser Region in Antiqua-Schrift. Es schließt sich eine serielle Einblendung nicht nur des Städtenamens Washington, sondern auch der Geschichte verschiedener Eissorten an. Dann kehrt der Text wieder auf die Zeitebene des 18. Jahrhunderts zurück und zitiert aus dem Abkommen von William Penn mit den Delaware-Indianern, mit Formulierungen, die in einem scharfen Kontrast zum Abschlachten der Indianer im oberen Teil der Seite stehen. Auch wenn beide Ereignisse direkt nichts miteinander zu tun haben, beleuchten sie sich doch wechselseitig: Es bleibt dem Lesepublikum überlassen, seine Schlüsse daraus zu ziehen. Schließlich folgt ein auf dieser Doppelseite nicht mehr beendetes Zitat von Benjamin Franklin, das den Einwanderern in die USA auf Kosten der Indianer eine goldene Zukunft verspricht. Die Kosten dieses Goldes stehen der Leserschaft vor Augen - Mobile ist folglich keineswegs ein unkritisches experimentelles Spiel mit Lexemen, das die großen Mythen der USA einfach wiedergäbe!

So werden durch die Einblendung unterschiedlichster Zitate Fragmente USamerikanischer Geschichte zusammengestellt, wobei die semantische Dimension der Verknüpfung dieser aus verschiedensten Bereichen zusammengetragenen 
Zitate fast gänzlich der Leserschaft zur Ausführung überantwortet wird. Wenige Jahre später sollte Julia Kristeva in einer Reihe bemerkenswerter Aufsätze in Anschluss an Michail Bachtin die Problematik der Intertextualität und eine neue intertextuelle Terminologie entwickeln, wobei ihr Hauptziel nicht in erster Linie das Zusammenwirken verschiedenster Textteile, sondern die Ausschaltung des in der abendländischen Philosophie dominierenden Subjektbegriffs war. Denn für Kristeva sollte an die Stelle der Intersubjektivität die Begrifflichkeit der Intertextualität treten.

Genau dies können wir in Mobile bereits erleben. Denn wer spricht hier eigentlich? Welche ist die Erzählerinstanz? Gibt es überhaupt eine Erzählerfigur? Nun, diese ist in der Tat auf eine minimale Schwundstufe gesunken: Sie ist zu einer leeren Figur verblasst, deren Ort noch markiert ist, die selbst aber nicht mehr als Trägerin einer sinnstiftenden Subjektivität verstanden werden kann. Julia Kristeva sollte dies theoretisch noch radikalisieren und in einem Aufsatz mit dem schönen Titel La productivité dite texte die Text- und Gewebemetaphorik so weit dehnen, dass das Subjekt aus diesem Gewebe verschwindet, dass es - mit anderen Worten - keines Autors und keiner Autorin mehr bedarf, um das Funktionieren des Textgewebes sicherzustellen. Der Text produziert und reproduziert sich gleichsam selbst.

Die Kehrseite der Medaille, die Ausschaltung des Autor-Subjekts, die 1967 explizit in Roland Barthes' breit rezipierten Aufsatz La mort de l'auteur (Der Tod des Autors) benannt wird, ist dabei - so der französische Zeichentheoretiker am Ende seines Aufsatzes - die Geburt des Lesers. Es wird ab sofort der Fähigkeit der Leserschaft anheimgestellt, nicht einen, sondern viele Dimensionen von Sinn in Texte zu legen: immer wieder von neuem den Versuch zu unternehmen, dass die Texte ,Sinn machen‘. Dass diese Paradoxie wiederum über kurz oder lang zur Subjektivität der Leserschaft zurückführen musste, erscheint aus heutiger Sicht geradezu zwangsläufig. Diese Tatsache ändert aber nichts daran, dass eine auf jegliches Subjekt verzichtende Textualitätsvorstellung seit den sechziger Jahren ungeheuer produktiv wurde. Michel Butors Mobile zwingt jedenfalls durch seine ständige Beweglichkeit und die immer wieder eingeschalteten Auszüge und Zitate anderer Werke zu einer völligen Neukonzeption dessen, was ein literarisches Werk zu sein hat. Auch in theoretischer Hinsicht hat dieser Text von 1962 ungeheuer anregend, ja in seiner Zeit sogar aufregend gewirkt.

Am Ende unserer Beschäftigung mit Michel Butors Mobile möchte ich wie angekündigt eine letzte Doppelseite folgen lassen, die sich wenige Seiten nach der zuvor analysierten situiert: 
Vous avez soif? Buvez Coca-Cola! - Ou le costume de Casper, l'aimable fantôme " qui ne veut point vous effrayer, mais simplement être un ami. Suaire de rayonne blanche avec capuchon permettant de cacher son identité, garniture rouge et noire ".

MONROE, dans le damier du comté de Turner comme

MARION, DAKOTA DU SUD.

Le reflet cramoisi sur les eaux.

La rivière Vermillon qui se jette dans le Missouri.

Un couple d'oies du Canada devant une touffe de roseaux, le jars dressé, retournant sa tête en arrière, noire avec la gorge blanche, le bec noir entrouvert laissant voir la langue rose pointue.

\section{MOUNT VERNON.}

MILFORD, État de l'œil d'épervier.

En attendant ce triomphal retour, ne fallait-il point reconstituer autour de soi une nouvelle Europe, effacer le plus possible de son esprit ce continent qui nous accueillait mais nous effrayait?

\begin{tabular}{|c|c|c|c|}
\hline \multirow{3}{*}{ Nouvelle } & \multirow[b]{2}{*}{ Nouvelle } & Nouzille & \multirow{2}{*}{ France, } \\
\hline & & Angleterre, & \\
\hline & Écosse, & & \multirow{4}{*}{$\begin{array}{l}\text { York, } \\
\text { Hollande, }\end{array}$} \\
\hline Nouveau & Brunswick, & & \\
\hline \multirow[b]{4}{*}{ Nouvelle } & \multirow[b]{3}{*}{ Nouvelle } & Nouveau & \\
\hline & & Nouvelle & \\
\hline & & Suède, & \\
\hline & Orléans, & & \\
\hline \multirow[t]{3}{*}{ Nouveau } & & & \\
\hline & & Nouveau & fersey, \\
\hline & & Nouvelle & Amsterdam \\
\hline
\end{tabular}

Mobile 159

Abb. 105d: Butor, Michel: Mobile, 1962, S. 159.

Bei dieser Seite sind beide Hälften des gesamten Blattes grafisch unterschiedlich genutzt. Die erste Hälfte der Seite beginnt mit einem Beispiel von Massenkommunikation, genauer: mit einer Passage US-amerikanischer Werbung, bei der selbstverständlich auch die Ikone US-amerikanischer Identitätsbildung, CocaCola, nicht fehlen darf. Dann folgt der schöne Ortsname Monroe, der sicher bei jedem Leser (und damit ist diesmal vorwiegend der männliche gemeint) eben- 
falls Erinnerungen an eine berühmte Ikone weckt, die schöne Marylin. Danach schließt sich in Kursivschrift eine lyrisch-literarische Sicht eines Naturphänomens an, gefolgt von in Antiqua-Schrift gesetzten Äußerungen zu Hydrologie (also vor allem zu den Flüssen) und Fauna des nordamerikanischen Raums, unter Einschluss natürlich der ornithologischen Besonderheiten.

Auf die Erwähnung von Mount Vernon, dem heute musealen ehemaligen Landsitz des ersten Präsidenten der Vereinigten Staaten, George Washington, folgt eine serielle Vermischung von Ortsnamen und - anstelle der Bezeichnung des Staates - ornithologischen Informationen. Schließlich stößt man auf der ersten Halbseite auf eine rhetorische Frage, die das Gefühl der europäischen Einwanderer zu reflektieren sucht, die der amerikanische Kontinent zwar aufnahm, denen er aber zugleich ordentlich Angst einflößte. Zur Beruhigung dieses Gefühls listet der „Nouveau romancier“ Michel Butor all die mit dem Adjektiv „neu“ versehenen Orts-, Landschafts- und Staatennamen auf. So entsteht eine Art serielles Bild-Gedicht, das aus einer Reihe derartiger Wortzusammensetzungen besteht. Dabei wird gerade im Kontrast zu der zuvor besprochenen Doppelseite deutlich, wie sehr dieses ,Neue‘ ein ,Altes“ ausgrenzt, tötet und überdeckt. Hier handelt es sich nicht um das alte Europäische, das gleichzeitig in diesen Ortsnamen erscheint, sondern um das Indianische, das zum Schweigen verurteilt ist, ganz wie die Kulturen und Völker, die ursprünglich den amerikanischen Kontinent bewohnten, aus der Erinnerung der siegreichen Weißen geschwunden und verschwunden sind. Die verschiedenen Konfigurationen derselben Serien erzielen in jedem einzelnen Fragment wieder neue Zusammenhänge und Verbindungen, die zwar von den Lesenden geschaffen werden müssen, von Mobile aber als Potentialität oder Virtualität in Buchform präsent gehalten werden. Es kommt auf die Leserin und den Leser an ...

Nach unserem Durchgang herrscht kein Zweifel: Mobile ist ein Experimentaltext, vielleicht sogar eine „œuvre-limite“, ein Grenztext! Das erzählende Ich samt seiner moralischen Kategorien ist aus dem Text verschwunden, auch wenn fraglos noch eine Schwundstufe übriggelassen wurde, um die ehemalige Position dieses Ich zu markieren. Alles wird in diesen Textfetzen auf dieselbe Stufe gestellt, gleichgültig, ob es sich um Gesprächs- oder Vertragsfetzen, um literarische oder ornithologische Fragmente, um Biographeme eines Reisenden oder Namenskataloge handelt. Der Text führt vor, wie sich alles zusätzlich und vieldeutig semantisieren lässt, wie selbst die unscheinbarsten Textfragmente weitere Bedeutungsebenen als Folge jener Mobilität der einzelnen Teile erhalten, die dadurch jeden Teil mit allen anderen in Kontakt und Kommunikation setzen. Alles ist mit allem verbunden: Alles ist Wechselwirkung.

So entsteht ein geradezu unendlicher Text, auch wenn er nach gut fünfhundert Seiten endet. Es handelt sich um ein Buch, wie Maurice Blanchot es 
in seinem Le livre à venir für die Zukunft kommen sah und wie es bestimmten Theoremen von Jorge Luis Borges entspricht, wenn es auch gänzlich andere literarische Formen annahm, als sie der Argentinier erdacht hätte. Alle Gattungen werden repräsentiert, und alle Gattungen werden letztlich von Butor (oder dem Text) experimentellen Sprachspielen unterworfen. Mobile ist ein in unablässige Bewegung gesetzter Text, der darauf wartet, dass jede Leserin und jeder Leser ihn ihrerseits in zusätzliche Bewegungen versetzen. So ist Mobile vielleicht doch ein Text der Reiseliteratur und die ständige Mobilität seine unbestreitbare Grundregel. Der Text berichtet von einer Reise, die viele Reisen ist; vor allem aber provoziert er weitere Reisen der Lesenden selbst. In seiner radikalen Offenheit handelt es sich hier um ein Stück Reiseliteratur im Zeichen einer Postmoderne, welche ihren offenen Kunstcharakter zelebriert. 